\title{
Comparison of the clinical effectiveness between the streamlined liner of pharyngeal airway (SLIPA) and the laryngeal mask airway by novice personnel
}

\author{
Seok-Kyeong Oh, Byung Gun Lim, Heezoo Kim, and Sang Ho Lim \\ Department of Anesthesiology and Pain Medicine, Korea University Guro Hospital, Seoul, Korea
}

Background: The aim of this study was to compare the streamlined liner of the pharynx airway (SLIPA) with the classic laryngeal mask airway when used by novice personnel.

Methods: There were 114 patients enrolled into this study who underwent general anesthesia were randomly allocated into one of 2 groups; LMA group $(n=57)$ or SLIPA group $(n=57)$. After insertion, insertion success rate, insertion time, and hemodynamic responses to insertion were accessed. After surgery, postoperative airway morbidity (sore throat, dysphonia, dysphagia) were evaluated.

Results: The SLIPA was successfully inserted in $96 \%$ of patients $(55 / 57)$ and the LMA in $93 \%(53 / 57)(\mathrm{P}=0.408)$. First attempt success rates were $88 \%(44 / 57)$ and $77 \%(50 / 57)$ in the SLIPA and the LMA $(\mathrm{P}=0.142)$. The successful insertion time in SLIPA group $(33.4 \pm 11.0 \mathrm{sec})$ was significantly shorter than that of LMA group $(38.8 \pm 16.6 \mathrm{sec})$ (P $=0.048)$ and the insertion time at the first attempt was also shorter in SLIPA group (31.0 $\pm 6.3 \mathrm{sec})$ than LMA group $(34.7 \pm 8.6 \mathrm{sec})(\mathrm{P}=0.013)$. There was no statistically significant difference between the two groups in hemodynamic responses and postoperative airway morbidity.

Conclusions: The SLIPA was similar to the LMA in insertion success rate, hemodynamic response, and postoperative airway morbidity by novice personnel. The insertion time at the first attempt and successful insertion time of the SLIPA were significantly shorter than those of the LMA. Therefore, the SLIPA could be a useful alternative to the LMA as primary SGA for novice personnel. (Korean J Anesthesiol 2012; 63: 136-141)

Key Words: Airway, Laryngeal mask, SLIPA, Supraglottic.

Received: February 7, 2012. Revised: March 9, 2012. Accepted: March 21, 2012.

Corresponding author: Sang Ho Lim, M.D., Ph.D., Department of Anesthesiology and Pain Medicine, Korea University Guro Hospital, 80, Gurodong, Guro-gu, Seoul 152-703, Korea. Tel: 82-2-2626-1437, Fax: 82-2-851-9897, E-mail: Lsh1102@unitel.co.kr

(c) This is an open-access article distributed under the terms of the Creative Commons Attribution Non-Commercial License (http:// creativecommons.org/licenses/by-nc/3.0/), which permits unrestricted non-commercial use, distribution, and reproduction in any medium, provided the original work is properly cited. 


\section{Introduction}

Many supraglottic airway devices (SGAs) have been developed and widely used for mechanically ventilated patients during surgery and as a valuable rescue airway tool in emergency airway management, since the LMA (laryngeal mask airway, LMA classic ${ }^{\mathrm{TM}}$, LMA North America Inc., San Diego, USA) was developed and introduced in the early 1980s by Brain [1].

The SLIPA (streamlined liner of the pharyngeal airway, SLIPA $^{\mathrm{TM}}$, SLIPA Medical Ltd., London, UK) is a relatively new, single-use SGA made of soft plastic. The SLIPA is regarded to be more advantageous than LMA, because it provides a higher oropharyngeal leak pressure, better protection from regurgitated liquid and less perilaryngeal gas leakage [2-4]. In addition, it requires fewer steps than LMA for placement because it can be inserted without cuff inflation or a biteblock, therefore making SLIPA easier to insert than the LMA by novice personnel [5]. However, there are few reports about the usefulness of SLIPA as a primary SGA for novice personnel during surgery under general anesthesia. The purpose of this study was to compare the SLIPA with the LMA in paralyzed and anesthetized patients in regard to insertion success rate, insertion time, hemodynamic responses to insertion, and postoperative airway morbidity such as sore throat, dysphagia, and dysphonia when used by novice personnel.

\section{Materials and Methods}

The study protocol was approved by the Institutional Review Board (MD11017) and written informed consent was obtained from each patient. One-hundred fourteen patients aged 1865 years and ASA physical status I-II, who were scheduled for elective surgery to be conducted under general anesthesia, who were enrolled into this study. Exclusion criteria were patients with morbid obesity (BMI > 30), upper respiratory symptoms, restricted mouth opening below $2.5 \mathrm{~cm}$, patients who were presented for head and neck surgery or thoracic surgery, patients at risk of pulmonary aspiration, and patients electing for surgery in the non-supine position.

In the present study, 'novice' personnel are defined as someone who has previously used any kind of LMA or SLIPA less than 5 times. All of the 'novice' personnel are residents in the department of anesthesiology and pain medicine. They received education according to the manufacturers' instructions and watched videos on inserting the devices and learned by the demonstration of the principal investigator inserting each device 3 times. The insertion of assigned SGA was performed under the observation of the principal investigator. The principal investigator had 5 years experience with using the LMA and had used the SLIPA more than 50 times before this study. Only 3 attempts at insertion were allowed for each practitioner; otherwise they would not qualify as a novice for the purpose of this study. Nineteen residents enrolled and each practitioner inserted each of the 2 devices 3 times, therefore a total of 114 patients participated in this study.

We randomized the patients into the LMA group (group $\mathrm{L}, \mathrm{n}=57$ ) and the SLIPA group (group $\mathrm{S}, \mathrm{n}=57$ ) based on the random allocation rule as a kind of restricted randomization [6]. The principle investigator prepared 57 cards for each group, 3 for each of the practitioners. Cards were labeled from S1 to S19 and from L1 to L19, 3 of each, and then the cards were inserted into envelops and shuffled. S1 means the number 1 practitioner would insert SLIPA and L19 means the number 19 practitioner would insert LMA. To reduce the selection bias of random allocation rule [7] as much as possible, the principal investigator did not let the practitioners know how many insertions would be performed with each device. Patient group and practitioner allocation was concealed until 5-10 minutes before induction of anesthesia by the means of sealed envelopes. There was no difference between the 2 groups in terms of demographics (gender, age, weight, height) and anesthetic time (Table 1).

All patients received glycopyrrolate $0.2 \mathrm{mg}$ and midazolam $2 \mathrm{mg}$ intramuscularly about 30 minutes before induction as a premedication and were monitored by electrocardiography, non-invasive blood pressure and pulse oximetry in the operating room. Following preoxygenation for three minutes, anesthesia was induced by intravenous administration of thiopental sodium $5 \mathrm{mg} / \mathrm{kg}$. After the loss of eyelash reflex and respiration, manual ventilation was started with oxygen at 8 $\mathrm{L} / \mathrm{min}$. Then manual ventilation was maintained with $100 \%$ $\mathrm{O}_{2}$ and $2.5 \%$ Sevoflurane at $8 \mathrm{~L} / \mathrm{min}$ for 2 minutes after the injection of rocuronium $0.6 \mathrm{mg} / \mathrm{kg}$. After confirming absence of motor response to jaw thrust, the assigned SGA was inserted into each patient. A jaw thrust was performed by the principal investigator to facilitate the atraumatic insertion. The size of the device was selected by the principal investigator based on the manufactures' guidelines. The size of LMA classic was

Table 1. Demographic and Clinical Data

\begin{tabular}{lcc}
\hline & $\begin{array}{c}\text { Group L } \\
(\mathrm{n}=57)\end{array}$ & $\begin{array}{c}\text { Group S } \\
(\mathrm{n}=57)\end{array}$ \\
\hline Age (yr) & $38.1 \pm 14$ & $41.8 \pm 14$ \\
Sex (M/F) & $21 / 36$ & $23 / 34$ \\
Weight (kg) & $57.2 \pm 12$ & $61.6 \pm 9.5$ \\
Height (cm) & $160.5 \pm 8.3$ & $162.8 \pm 6.8$ \\
Thyromental distance (mm) & $63.4 \pm 6.1$ & $62.6 \pm 5.5$ \\
Mallampati score (I/II/III) & $21 / 33 / 3$ & $24 / 30 / 3$ \\
Duration of anesthesia (min) & $106.7 \pm 67$ & $109.6 \pm 50$ \\
\hline
\end{tabular}

Values are expressed as mean \pm SD or number. There were no statistical differences between groups. 
chosen based upon body weight; size 3 if the patient weighed under $50 \mathrm{~kg}$, size 4 between $50-70 \mathrm{~kg}$ or size 5 over $70 \mathrm{~kg}$. The SLIPA size was chosen by matching the width across the thyroid cartilage with that of the bridge of the SLIPA [2]. Water-soluble lubricant was applied to both devices. A pillow of $5-10 \mathrm{~cm}$ was positioned beneath each patient's head with the neck flexed and head extended. In group L, the cuff was fully deflated, held like a pen, and inserted while pressing against the palatophayngeal curve using the index finger [1]. In group S, the lubricated SLIPA was inserted with the bridge area collapsed by hand while lifting the patient's jaw with the other hand and pushed past the base of the tongue and clipped into position with the heel located in the nasopharynx according to the manufacturers' instructions.

After inserting the device, we confirmed absence of gas leakage in the mouth under peak inspiratory airway pressure of $20 \mathrm{cmH}_{2} \mathrm{O}$ during positive ventilation, breathing heard equally in both lungs, normal square waved capnography and absence of audible sound during epigastric auscultation. When all these criteria were satisfied, we determined that the insertion was successful. If the device was not advanced into the pharynx or not ventilated well due to airway obstruction or considerable air leakage through the oral cavity or esophagus was observed, we determined that the insertion failed and finished measuring the insertion time. If the first attempt at inserting the device was unsuccessful, manual ventilation was resumed with $100 \%$ oxygen and $2.5 \%$ sevoflurane at $8 \mathrm{~L} / \mathrm{min}$ for 2 minutes to allow one additional attempt. If the second attempt was unsuccessful, it was recorded as an insertion failure and endotracheal intubation was performed.

When the insertion was performed, the insertion time was recorded. Insertion time was measured from the opening of the patient's mouth by the practitioner to when the first satisfactory breath of at least $7 \mathrm{ml} / \mathrm{kg}$ was obtained. The successful insertion time (the time to provide an effective airway) was the sum of the time taken for insertion at the first attempt and time taken for insertion at the second attempt until the insertion was successful excepting the manual ventilation time of 2 minutes. To compare the hemodynamic response to each device, heart rate (HR), systolic blood pressure (SBP), and diastolic blood pressure (DBP) were recorded at the baseline, just before insertion and just after insertion.

For all patients in whom successful insertion of SGAs was achieved, mechanical ventilation was started with tidal volume of $8-10 \mathrm{ml} / \mathrm{kg}$, and respiratory rate of $10-14 \mathrm{breaths} / \mathrm{min}$ to achieve end-tidal carbon dioxide concentration of $30-40$ $\mathrm{mmHg}$. Anesthesia was maintained with nitrous oxide $\left(\mathrm{N}_{2} \mathrm{O}\right)$ $1.5 \mathrm{~L} / \mathrm{min}$, oxygen $1.5 \mathrm{~L} / \mathrm{min}$ and sevoflurane $1.5-2.5 \%$, and rocuronium was administered as required to maintain adequate surgical relaxation. In group $\mathrm{L}$, the cuff pressure was set to below $60 \mathrm{cmH}_{2} \mathrm{O}$ to reduce the risk of mucosal ischemia and nerve injury. At the end of surgery, $100 \%$ oxygen was supplied, and glycopyrrolate $0.4 \mathrm{mg}$ and pyridostigmine $10 \mathrm{mg}$ were injected intravenously to reverse neuromuscular block. When spontaneous respiration was established and the patient was able to obey command, all SGAs were removed in the operating room before transfer to the recovery room.

To assess postoperative airway morbidity, the patients were queried for sore throat, dysphagia, and dysphonia, both before they were transferred to the general ward from the recovery room, and after 24 hours. Sore throat was defined as pain or irritation in the throat at rest, dysphagia as pain or irritation when swallowing and dysphonia, as pain or irritation when vocalizing.

SPSS 17.0 was used for statistical analysis, Chi-square or Fisher's exact test were used for comparisons of two groups for the success rate and postoperative airway morbidity and unpaired t-test or Mann-Whitney rank sum test were used for the insertion times and hemodynamic data between the two groups. One Way Repeated Measures Analysis of Variance was used for comparisons of hemodynamic data within each group and Tukey test were used as post-hoc test. P values $<0.05$ were considered statistically significant. Sample size calculation was done by G* Power (version 3.0.1, Germany). Through the preliminary tests of 5 patients of each group, mean successful insertion time was calculated as $35.2 \pm 8.5 \mathrm{sec}$ in group L, $30 \pm 7.1$ sec in group S. The total sample size was set as 114 (57 per each group) calculated from an effect size of 0.66 , a power of $95 \%$, and $\alpha$ error of 0.05 .

\section{Results}

The SLIPA was successfully inserted in $96 \%$ of patients (55/57) and the LMA in $93 \%(53 / 57)(\mathrm{P}=0.408)$. First attempt success rates were $88 \%(44 / 57)$ and $77 \%(50 / 57)$ in the SLIPA and the LMA, but the difference was not statistically significant ( $\mathrm{P}=$ 0.142). The successful insertion time in group $S(33.4 \pm 11.0 \mathrm{sec})$ was significantly shorter than group L $(38.8 \pm 16.6 \mathrm{sec})(\mathrm{P}=0.048)$

Table 2. Insertion Success Rate and Insertion Time

\begin{tabular}{lll}
\hline & $\begin{array}{l}\text { Group L } \\
(\mathrm{n}=57)\end{array}$ & $\begin{array}{l}\text { Group S } \\
(\mathrm{n}=57)\end{array}$ \\
\hline $\begin{array}{ll}\text { Insertion success rate (\%) } \\
\text { First attempt }\end{array}$ & $77.2 \%(44 / 57)$ & $87.7 \%(50 / 57)$ \\
Second attempt & $69.2 \%(9 / 13)$ & $71.4 \%(5 / 7)$ \\
$\quad$ Overall & $92.9 \%(53 / 57)$ & $96.4 \%(55 / 57)$ \\
Insertion time (sec) & & \\
$\quad$ Insertion time at the first attempt & $34.7 \pm 8.6$ & $31.0 \pm 6.3^{*}$ \\
$\quad$ Successful insertion time & $38.8 \pm 16.6$ & $33.4 \pm 11.0 *$ \\
\hline
\end{tabular}

Values are expressed as mean \pm SD or number. Group L: LMA group. Group S: SLIPA group. *P $<0.05$ compared with group L. 
A

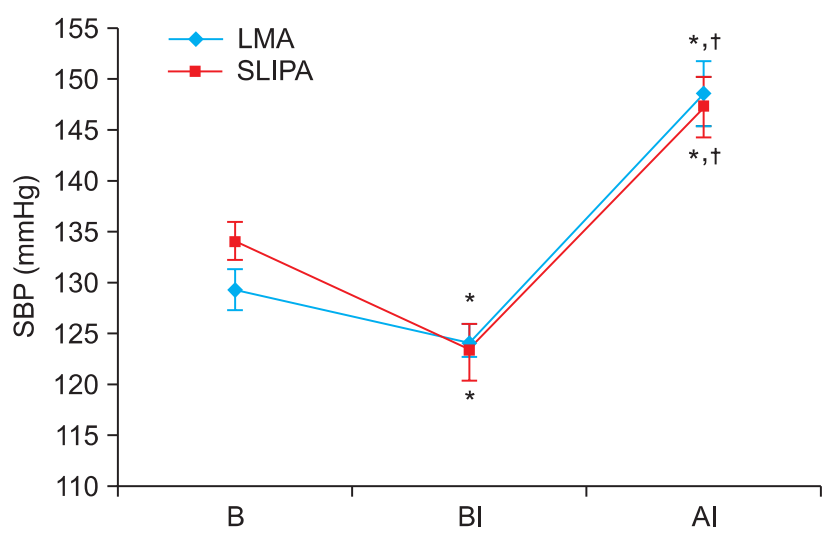

C

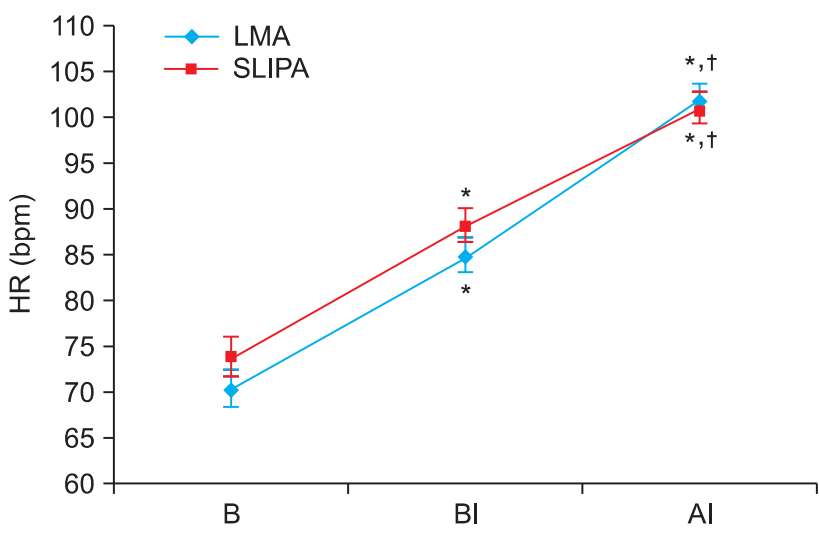

and the insertion time at the first attempt was also significantly shorter in group S $(31.0 \pm 6.3 \mathrm{sec})$ than in group L $(34.7 \pm 8.6$ sec) $(\mathrm{P}=0.013)$ (Table 2). The hemodynamic changes in each group showed the increase in HR, SBP, DBP just after insertion compared to just before insertion (Fig. $1, \mathrm{P}<0.05$ ). There was no statistically significant difference between the two groups in the hemodynamic responses (Fig. 1) and postoperative airway morbidity (sore throat, dysphagia, and dysphonia) (Table 3). There was no regurgitation of gastric contents or aspiration in the 2 groups.

There were 4 failures in group L. In 2 of these cases, the tip of the LMA cuff was folded. In the first case, the novice failed but the principal investigator was able to easily insert the device. In the second case, the principal investigator was also unable to insert the device, and endotracheal intubation was then performed. In the third case, failure was a result of oral bleeding. The fourth patient developed a perilaryngeal leak despite successful insertion. In addition, endotracheal intubation was performed in the last 2 cases.

There were 2 failures of SLIPA application. In one patient, a leak occurred even though the SLIPA was inserted easily. It was replaced with a SLIPA of larger size that ventilated well, which
B

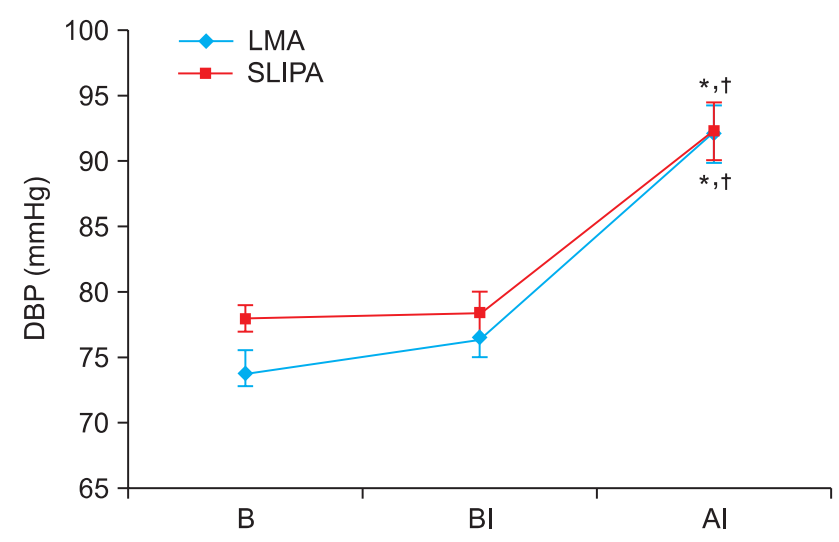

Fig. 1. Hemodynamic response to insertion. (A) Systolic blood pressure, (B) Diastolic blood pressure, (C) Heart rate. B: Baseline, BI: Before-insertion, AI: After-insertion. Data are expressed as mean \pm SEM, There were no statistical differences between groups. ${ }^{*} \mathrm{P}<0.05$ compared with baseline value, ${ }^{\dagger} \mathrm{P}<0.05$ compared with Beforeinsertion value.

Table 3. Incidences of Postoperative Airway Morbidity

\begin{tabular}{lrrrrr}
\hline & \multicolumn{2}{c}{$\begin{array}{c}\text { Group L } \\
(\mathrm{n}=57)\end{array}$} & & \multicolumn{2}{c}{$\begin{array}{c}\text { Group S } \\
(\mathrm{n}=57)\end{array}$} \\
\cline { 2 - 5 } \cline { 4 - 6 } & $\mathrm{RR}$ & $24 \mathrm{hr}$ & & $\mathrm{RR}$ & $24 \mathrm{hr}$ \\
\hline Sore throat & 19 & 13 & & 17 & 9 \\
Dysphonia & 20 & 16 & & 21 & 11 \\
Dysphagia & 12 & 9 & 12 & 6 \\
\hline
\end{tabular}

Values are expressed as number. There were no statistical differences between groups. RR: recovery room. $24 \mathrm{hr}: 24$ hours after the end of surgery.

constitutes a failure of selecting an appropriate size. In the second patient, 2 different sizes of SLIPA failed to be insert, so endotracheal intubation was performed.

\section{Discussion}

SGA, which is easier to learn how to use than an endotracheal tube, has proved extremely useful in managing a difficult airway or during emergency resuscitation [8]. For these reasons SGAs are very useful for airway management when used by personnel 
who are unfamiliar with endotracheal intubation, but there are few reports about the usefulness of SLIPA as primary SGA for novice personnel during surgery under general anesthesia. Therefore, we focused particularly on the performance and efficacy of SLIPA by novice personnel in the present study. McCrirrick et al. [9] defined the 'novice' airway provider as someone who has previously used LMA less than 15 times. However, in the present study, 'novice' personnel are who has previously used any kind of LMA or SLIPA less than 5 times, because there was a short learning curve of about 13 cases for insertion of SLIPA in a previous study [10].

The performance and efficacy of SLIPA when used by novice personnel were reported in the following studies. Hein et al. [5] noted that first attempt success rates were 67\% (24/36) and $83 \%(30 / 36)$ in the Soft Seal LMA (SS-LM) and the SLIPA by medical student with no experience of airway management. Choi et al. [11] noted that first attempt success rates were 93.3\% and $73.3 \%$ in the ProSeal LMA (PLMA) and SLIPA respectively by a practitioner with PLMA experience, but little experience with SLIPA. Compared to PLMA insertion, SLIPA insertion took longer and was more difficult due to the relative lack of experience with the device and difficulty of selecting its appropriate size. However, in the present study, this difficulty was mitigated by having principal investigator choose the proper size.

In this study, first attempt success rates were $77.2 \%$ and 87.7\% in the LMA classic and SLIPA and overall success rates were $92.9 \%$ and $96.4 \%$. These results are similar to those found by Hein et al. [5], but slightly higher in both devices. We assume that the reason for the different results in these studies can be attributed for the difference in application method of SGA by medical students in the previous study had never used or been trained to use any airway device, whereas the residents in this study have been trained in the airway management despite lack of experience in using the LMA and SLIPA.

The insertion time at the first attempt, which represents the subjective ease of insertion, was significantly longer in group $\mathrm{L}$ than group S. The SLIPA, which has no cuff to inflate and fewer steps to remember for application than the LMA, might be easier to insert for novice personnel. Hein et al. [5] reported that the successful insertion time was significantly shorter with the SLIPA (40.6 sec) than with SS-LM (66.9 sec), and Tan et al. [12] found that the SS-LM took significantly longer to insert when compared with the LMA classic. From these 2 studies, we predicted that the difference in the insertion time between the LMA classic and SLIPA would be smaller than the difference between the SS-LM and SLIPA. In fact, in this study, successful insertion time was $38.8 \pm 16.6 \mathrm{sec}$ and $33.4 \pm 11.0 \mathrm{sec}$ in group $\mathrm{L}$ and group $S$ respectively and has a significant difference.

A previous report, which was performed by a single inves- tigator with LMA experience but no experience with SLIPA, has shown that SLIPA causes significantly higher BP response following insertion as compared to LMA [10]. Therefore we assumed that SLIPA, which is constructed of stiffer plastic material than the silicone of LMA, would cause more mucosal injury to the deeper pharyngeal structures and thus would cause higher BP and HR than LMA. However, in this study, there was no significant difference between the two groups in the hemodynamic response. The longer the insertion times of SGAs, the greater the risk of tachycardia and hypertension [13]. In the present study, the number of the second insertion required was greater in group $\mathrm{L}$ than in group $\mathrm{S}$ and the total insertion time took longer in group $\mathrm{L}$. Therefore, it is considered that the hemodynamic instability of SLIPA shown in the previous study [10] might be offset by the longer insertion time of LMA in this study.

Concerning postoperative airway morbidity, its incidences according to Miller and Camporota [2] were 57\%, 49\%, 30\% for an endotracheal tube, SLIPA, PLMA, respectively, and those according to Choi et al. [11] were $48 \%$ (14/29), 26\% (8/30) respectively for SLIPA and PLMA. Therefore, it seems to be the SLIPA has a relatively high incidence of sore throat. On the other hand, in the present study, a relatively high rate of sore throat in group L might have been due to the pressure exerted on the pharyngeal structures by the cuff of the LMA [14]. In addition, when $\mathrm{N}_{2} \mathrm{O}$ is used to maintain anesthesia, its diffusion into the cuff of the LMA increases the pressure on the pharyngeal structures. Therefore close monitoring of the cuff pressure of LMA and keeping it low are necessary when $\mathrm{N}_{2} \mathrm{O}$ is used, however SLIPA does not have a cuff, thus it is not affected by the use of $\mathrm{N}_{2} \mathrm{O}$. So this gives SLIPA an advantage over the use of LMA.

Airway management is of utmost importance in critical patients, for whom endotracheal intubation remains the mainstay. However, because it is a difficult skill to acquire and success rates in novices are unacceptably low, SGAs could become promising alternatives. Reinhart and Simmons [15] reported that LMA could be inserted more easily than endotracheal tube with shorter insertion time and higher success rate by novice personnel. The present study shows that novice personnel can use both LMA and SLIPA effectively after a short time of training, SLIPA especially has a shorter insertion time and similar success rate, hemodynamic changes, and postoperative airway morbidity as compared to LMA. In conclusion, the SLIPA could be a useful alternative to the LMA as primary SGA for novice personnel during surgery under general anesthesia.

\section{References}

1. Brain AI. The laryngeal mask-a new concept in airway management. Br J Anaesth 1983; 55: 801-5. 
2. Miller DM, Camporota L. Advantages of ProSeal and SLIPA airways over tracheal tubes for gynecological laparoscopies. Can J Anaesth 2006; 53: 188-93.

3. Woo YC, Cha SM, Kang H, Baek CW, Jung YH, Kim JY, et al. Less perilaryngeal gas leakage with SLIPA than with LMA- ProSeal in paralyzed patients. Can J Anaesth 2011; 58: 48-54.

4. Miller DM, Light D. Laboratory and clinical comparisons of the streamlined liner of the pharynx airway (SLIPA) with the laryngeal mask airway. Anaesthesia 2003; 58: 136-42.

5. Hein C, Owen H, Plummer J. Randomized comparison of the SLIPA (Streamlined Liner of the Pharynx Airway) and the SS-LM (Soft Seal Laryngeal Mask) by medical students. Emerg Med Australas 2006; 18: $478-83$.

6. Schulz KF, Grimes DA. Generation of allocation sequences in randomised trials: chance, not choice. Lancet 2002; 359: 515-9.

7. Lachin JM. Statistical properties of randomization in clinical trials. Control Clin Trials 1988; 9: 289-311.

8. Wilkins CJ, Cramp PG, Staples J, Stevens WC. Comparison of the anesthetic requirement for tolerance of laryngeal mask airway and endotracheal tube. Anesth Analg 1992; 75: 794-7.

9. McCrirrick A, Ramage DT, Pracilio JA, Hickman JA. Experience with the laryngeal mask airway in two hundred patients. Anaesth Intensive Care 1991; 19: 256-60.

10. Puri GD, Hegde HV, Jayant A, Bhukal I. Haemodynamic and Bis- pectral index response to insertion of the Streamlined Liner of the Pharynx Airway (SLIPA): comparison with the laryngeal mask airway. Anaesth Intensive Care 2008; 36: 404-10.

11. Choi YM, Cha SM, Kang H, Baek CW, Jung YH, Woo YC, et al. The clinical effectiveness of the streamlined liner of pharyngeal airway $\left(\mathrm{SLIPA}^{\mathrm{TM}}\right)$ compared with the laryngeal mask airway ProSeal ${ }^{\mathrm{TM}}$ during general anesthesia. Korean J Anesthesiol 2010; 58: 450-7.

12. Tan MG, Chin ER, Kong CS, Chan YH, Ip-Yam PC. Comparison of the re-usable LMA Classic and two single-use laryngeal masks (LMA Unique and SoftSeal) in airway management by novice personnel. Anaesth Intensive Care 2005; 33: 739-43.

13. Kim HS, Nam HW, Kim YH. The maximal changes of signs to laryngeal mask airway proseal ${ }^{\mathrm{TM}}$ Insertion or tracheal intubation after anesthetic induction in hypertensive patients. Korean J Anesthesiol 2002; 43: 145-9.

14. Brimacombe J, Holyoake L, Keller C, Barry J, Mecklem D, Blinco A, et al. Emergence characteristics and postoperative laryngopharyngeal morbidity with the laryngeal mask airway: a comparison of high versus low initial cuff volume. Anaesthesia 2000; 55 : 338-43.

15. Reinhart DJ, Simmons G. Comparison of placement of the laryngeal mask airway with endotracheal tube by paramedics and respiratory therapists. Ann Emerg Med 1994; 24: 260-3. 